\title{
Antibacterial activity of six medicinal Cameroonian plants against Gram-positive and Gram-negative multidrug resistant phenotypes
}

Igor K. Voukeng ${ }^{1}$, Veronique P. Beng ${ }^{2}$ and Victor Kuete ${ }^{1 *}$

\begin{abstract}
Background: Infectious diseases due to multidrug-resistant bacteria are one of the causes of treatment failures contributing to an increase in mortality and/or morbidity. In this study, we evaluated the antibacterial potential of different parts of six medicinal plants namely Alstonia boonei, Ageratum conyzoides, Croton macrostachys, Cassia obtusifolia, Catharanthus roseus and Paullinia pinnata against a panel of 36 multi-drug resistant (MDR) Gramnegative and Gram-positive bacteria.

Methods: Minimum Inhibitory Concentration (MIC) and Minimal Bactericidal Concentration (MBC) of the methanol extracts from different parts of the plants were determined using broth microdilution method; standard phytochemical methods were used for phytochemical screening.

Results: Several phytochemical classes such as polyphenols, sterols, triterpenes, alkaloids, flavonoids and saponins were identified in the plant extracts. MIC values obtained ranged from 64 to $1024 \mu \mathrm{g} / \mathrm{mL}$. Leaves extract of Catharanthus roseus (86.11\%), Croton macrostachys (83.33 \%) and Paullinia pinnata (80.55\%) displayed the best antibacterial spectra. The lowest MIC value of $64 \mu \mathrm{g} / \mathrm{mL}$ was obtained with the Paullinia pinnata stems extract and Cassia obtusifolia extract against the strain of Staphylococcus aureus MRSA8. Results also showed that the tested samples generally displayed bacteriostatic effects with MBC values obtained in only $3.35 \%$ of the cases where plant extracts were active.

Conclusion: The results obtained at the end of this study demonstrate for the first time the antibacterial activity of the studied medicinal plants against MDR bacteria. The tested plants could be a reservoir of molecules to fight against MDR bacterial infections.
\end{abstract}

Keywords: Cameroon, Gram-negative bacteria, Gram-positive bacteria, Medicinal plant, Multi-drug resistance, Antibacterial activity

\section{Background}

Infectious diseases caused by multidrug-resistant bacteria are growing steadily and are associated with a significant attributable mortality $[1,2]$. The emergence of multi-drug resistant (MDR) phenotypes was first linked to nosocomial infections; but nowadays they are increasingly responsible for community infections and all pathogenic

\footnotetext{
* Correspondence: kuetevictor@yahoo.fr

'Department of Biochemistry, Faculty of Science, University of Dschang, P.O. Box 67, Dschang, Cameroon

Full list of author information is available at the end of the article
}

microorganisms are concerned. In Gram-negative bacteria, one of the mechanisms of resistance is the lowering of intracellular amount of antibacterial substances due to the presence of the resistance nodulation cell division (RND)-type efflux pumps. This phenomenon gives possibility to bacteria developing resistance to a wide range of antibiotics, as well as several biocides [3, 4]. Gram-positive bacteria are also a major cause of hospitalization; infections due to Staphylococcus aureus resistant to methicillin (MRSA) are a major health problem both in hospitals and community environments [5]. MRSA is responsible for 
80461 severe infections and causing the death of 11,285 patients annually in the United States [6]. One of the possible ways to overcome this phenomenon of multiresistance is the continual search for new antibacterial molecules active vis-à-vis of MDR bacteria. With regard to the broad diversity of their secondary metabolites, medicinal plants represent undeniable sources of antibacterial agents. According to WHO [7], $80 \%$ of people in Africa have used medicinal plants for their health care; it is also estimated that among medicines sold worldwide, $30 \%$ contain compounds derived from medicinal plants [8]. Several African medicinal plants previously investigated for biological potential showed good antibacterial activities. Some of them include Treculia obovoidea [9], Albizia adianthifolia Laportea ovalifolia [10], Alchornea cordifolia, Pennisetum purpureum [11]. In our continuous search of phytochemicals to combat MDR bacterial infections, we designed the present study to evaluate the antimicrobial potential of six Cameroonian medicinal plants namely Alstonia boonei, Catharanthus roseus, Ageratum conyzoides, Croton macrostachys, Cassia obtusifolia, and Paullinia pinnata vis-à-vis MDR Gram-negative and Gram-positive phenotypes.

\section{Methods}

\section{Plant materials and extraction}

Various parts of plant (Table 1) were collected from different regions in Cameroon during the month of February 2014. These include Alstonia boonei (leaves and bark), Catharanthus roseus (leaves and stem), Ageratum conyzoides (whole plant), Croton macrostachys (leaves), Cassia obtusifolia (whole plant), and Paullinia pinnata (leaves and stem). After drying, each part was powdered and soaked in methanol for $48 \mathrm{~h}$ at room temperature, and then filtered using Whatman filter paper $\mathrm{N}^{\circ} 1$. The filtrate obtain were concentrated at $50{ }^{\circ} \mathrm{C}$ under reduce pressure in a vacuum to obtain each plant extract.

\section{Preliminary phytochemical screenings}

The presence of alkaloids, triterpenes, sterols, flavonoids, polyphenols and saponins were screened according to the common phytochemical methods described by Harborne [12].

\section{Chemicals}

Chloramphenicol and ciprofloxacin (Sigma-Aldrich, St. Quentin Fallavier, France) were used as reference antibiotics meanwhile $p$-Iodonitrotetrazolium chloride (INT) was used as microbial growth indicator.

\section{Bacterial strains and culture media}

The studied microorganisms included ATCC (American Type Culture Collection) and MDR clinical strains of Gram-negative bacteria (Escherichia coli, Pseudomonas aeruginosa, Enterobacter aerogenes, Providencia stuartii, Klebsiella pneumoniae and Enterobacter cloacae) and Gram-positive bacteria (Staphyloccocus aureus). Their bacterial features are summarized in Table 2; they were maintained at $4{ }^{\circ} \mathrm{C}$ on McConkey agar and Mannitol Salt Agar (MSA) for Gram negative and Gram positive bacteria respectively, and sub-cultured on Mueller Hinton Agar (MHA) for $24 \mathrm{~h}$ before any test. Mueller Hinton Broth (MHB) was used for MIC and MBC determinations.

\section{INT colorimetric assay for MIC and MBC determinations} Minimal inhibitory concentrations (MIC) of different plant extracts were determined using broth microdilution method described by Kuete et al. [13] with some modifications [9]. Briefly, plant extracts, chloramphenicol and ciprofloxacin were dissolved in dimethylsufoxide (DMSO)-MHB (10:90) and $100 \mu \mathrm{L}$ each solution was added to a 96 wells microplate containing $\mathrm{MHB}$, then serially diluted two-fold, followed by adding of $100 \mu \mathrm{L}$ of inoculum prepared in MHB. The microplate was sealed and incubated for $18 \mathrm{~h}$ at $37{ }^{\circ} \mathrm{C}$. The final concentration of inoculum was $1.5 \times 10^{6} \mathrm{CFU} / \mathrm{mL}$ and less than $2.5 \%$ for DMSO in each well; Wells containing DMSO $2.5 \%$ and inoculums were used as negative control whereas chloramphenicol and ciprofloxacin consist of positive control. After $18 \mathrm{~h}$ incubation, $40 \mu \mathrm{L}$ of INT $(0.2 \mathrm{mg} / \mathrm{mL})$ was added to each well and re-incubated for $30 \mathrm{~min}$. MIC was defined as the lowest concentration of plant extract that inhibited bacterial growth.

The determination of $\mathrm{MBC}$ was made by introducing $150 \mu \mathrm{L}$ of MHB in each well of 96 well plate. Then $50 \mu \mathrm{L}$ of the well contents which did not show any growth after incubation during MIC assays was introduced in the aforesaid plate accordingly, and incubated at $37{ }^{\circ} \mathrm{C}$ for $48 \mathrm{~h}$. The MBC was defined as the lowest concentration of plant extract, which did not produce a color change after addition of INT as described previously.

\section{Results}

\section{Phytochemical composition}

The results of qualitative analysis (Table 3) showed that plant extracts contain various phytochemical classes of secondary metabolites. Polyphenols, triterpenes and saponins were present in all plant extracts except those from Cassia obtusifolia, Catharanthus roseus leaves and stem respectively.

\section{In vitro antibacterial effect of plant extract}

The methanol extracts from different parts of plants were tested on 36 bacterial strains including 7 Grampositive and 29 Gram-negative bacterial strains. As 
Table 1 Information on plant used in the present study

\begin{tabular}{|c|c|c|c|c|}
\hline $\begin{array}{l}\text { Plant family/Plant sample - } \\
\text { Herbarium voucher number }\end{array}$ & Traditional use & $\begin{array}{l}\text { Part used in } \\
\text { this study }\end{array}$ & Potential active compounds characterized & Previously screened activity \\
\hline $\begin{array}{l}\text { APOCYNACEAE/Alstonia } \\
\text { boonei De Wild - 43368/ } \\
\text { HNC }\end{array}$ & $\begin{array}{l}\text { Fever, painful micturition, insomnia, } \\
\text { chronic diarrhea, rheumatic pains, } \\
\text { anti-venom (snake bites), malaria, } \\
\text { diabetes, helminths, arthritis }[28,29] \text {. }\end{array}$ & Leaves, bark & $\begin{array}{l}\text { Echitamine, echitamidine, Voacangine, akuammidine, } \\
\mathrm{N} \text { - } a \text {-formylechitamidine, } \mathrm{N} \text { - } a \text {-formyl-12- } \\
\text { methoxyechitamidine [29]. }\end{array}$ & $\begin{array}{l}\text { Antimalarial, antioxidant, analgesic, } \\
\text { anti-inflammatory, antipyretic [30-32]. }\end{array}$ \\
\hline $\begin{array}{l}\text { APOCYNACEAE/Catharanthus } \\
\text { roseus L. - 5689/HNC. }\end{array}$ & $\begin{array}{l}\text { Bleeding arresting, diabetes, fever, } \\
\text { rheumatism, cancer }[20,33] \text {. }\end{array}$ & Leaves, stem & $\begin{array}{l}\text { Vincristine, vinblastine, benzoic acid, p-hydroxybenzoic acid, } \\
\text { salicylic acid, 2,3-dihydroxybenzoic acid, 2,5-dihydroxybenzoic } \\
\text { acid, 3,4-dihydroxybenzoic acid, 3,5-dihydroxybenzoic acid, } \\
\text { gallic acid, vanillic acid, chlorogenic acid, kaemferol } \\
\text { trisaccharides, Quercetin trisaccharides, Syringetin } \\
\text { glycosides [20, 34]. }\end{array}$ & $\begin{array}{l}\text { Wound-healing, antimicrobial, } \\
\text { hypoglycemic, antioxidant [18, 20, 33]. }\end{array}$ \\
\hline $\begin{array}{l}\text { ASTERACEAE/Ageratum } \\
\text { conyzoïdes Linn. - 19050/ } \\
\text { SFR-Cam }\end{array}$ & $\begin{array}{l}\text { Purgative, fever, ulcers, wound, mental, } \\
\text { infectious diseases, headaches, } \\
\text { anti-inflammatory, diarrhea [35, 36]. }\end{array}$ & Whole plant & $\begin{array}{l}\beta \text {-caryophyllene, precocene I, friedelin, Lycopsamine, echinatine, } \\
\beta \text {-sitosterol, stigmasterol, } 5 \text {-methoxynobiletin, linderoflavone } B \text {, } \\
\text { eupalestin, sabinene, } \alpha \text { and } \beta \text { pinene, } \beta \text {-phellandrene, } \\
\text { 1,8-cineole and limonene, ocimene, eugenol [35]. }\end{array}$ & $\begin{array}{l}\text { Antimicrobial, anticonvulsant, analgesic, } \\
\text { anti-inflammatory, antipyretic, insecticidal, } \\
\text { antioxidant, antiplasmodial, } \\
\text { cytotoxic }[35,37,38] \text {. }\end{array}$ \\
\hline $\begin{array}{l}\text { EUPHORBIACEAE/Croton } \\
\text { macrostachys Hochst. } \\
\text { - 40501/HNC }\end{array}$ & $\begin{array}{l}\text { Malaria, antidiabetic, purgative mastitis, } \\
\text { wounds, gastrointestinal Complications } \\
\text { [39-41]. }\end{array}$ & Leaves & $\begin{array}{l}\text { Neoclerodan-5,10-en-19,63;20,12-diolide; } 3 a \text {, } \\
\text { 19-Dihydroxytrachylobane; 3a,18,19-Trihydroxytrachylobane, } \\
\text { lupeol, lupenone, betulinic acid, 28-O-acetylbetulin, betulin, } \\
\text { lupeol acetate, zeorin, benzoic acid, methyl gallate, methyl } 2 \text {, } \\
\text { 4-dihydroxy-3,6-dimethylbenzoate, lichexanthone, } \beta \text {-sitosterol, } \\
\text { and } \beta \text {-sitosterol palmitate, stigmasterol, botulin, crotepoxide [42, 43]. }\end{array}$ & $\begin{array}{l}\text { Antimicrobial, antimalarial, } \\
\text { cytotoxic }[38,39,41] \text {. }\end{array}$ \\
\hline $\begin{array}{l}\text { FABACEAE/Cassia obtusifolia } \\
\text { L. - 39847/HNC }\end{array}$ & $\begin{array}{l}\text { Laxative, eye infections, diarrhea, } \\
\text { urinary tract infections, gingivitis, } \\
\text { fever, cough [25]. }\end{array}$ & Whole plant & 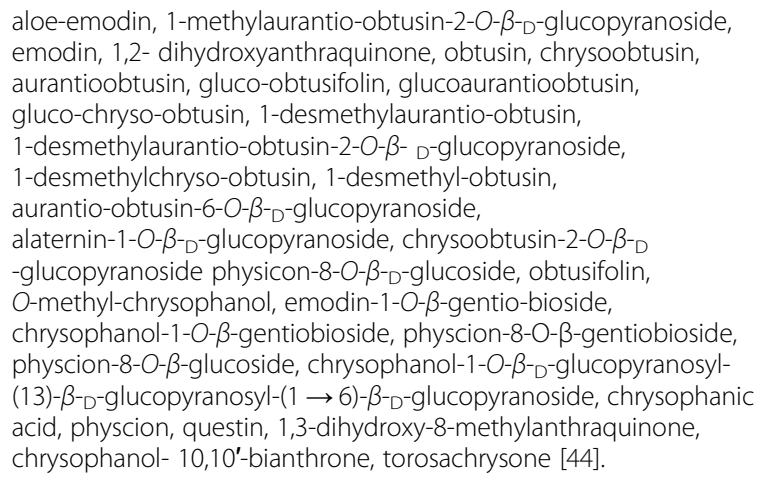 & $\begin{array}{l}\text { Antibacterial, antifungal, mosquito } \\
\text { larvicidal activity, platelet antiaggregatory, } \\
\text { neuroprotective }[25,45-47] \text {. }\end{array}$ \\
\hline $\begin{array}{l}\text { SAPINDACEAE/Paullinia } \\
\text { pinnata L. - 10702/ } \\
\text { SRF-Cam }\end{array}$ & Malaria, erectile dysfunction [24]. & Leaves, stem & $\begin{array}{l}\text { Paullinoside A, paullinomides A and B, } \beta \text {-amyrin, } 13 \beta \text {, } \\
17 \beta \text {-dihydroxy-28-norolean-12-ene, } \beta \text {-sitosterol glucopyranoside, } \\
\text { 2-O-methyl-L-chiro-inositol, L-quebrachitol, } \beta \text {-sitosterol, friedelin, } \\
\text { daucosterol, aridanin, lotoidoside }[24,48] \text {. }\end{array}$ & $\begin{array}{l}\text { Antiparasitic, antimicrobial, } \\
\text { cytotoxic }[24,38,49] \text {. }\end{array}$ \\
\hline
\end{tabular}

HNC Cameroon National Herbarium, SRF-Cam Société' des Réserves Forestières du Cameroun 
Table 2 Bacterial strains used in this study and their features

\begin{tabular}{|c|c|}
\hline Strains & Characteristics \\
\hline \multicolumn{2}{|l|}{ Escherichia coli } \\
\hline ATCC10536 & Reference strain \\
\hline AG100 & Wild-type E. coli K-12 \\
\hline AG100A & AG100 $\triangle a c r A B:: K^{\prime} N^{R}$ \\
\hline AG100A & $\begin{array}{l}\triangle a c r A B \text { mutant } A G 100, \text { with } \\
\text { over-expressing acrF gene ; TET }\end{array}$ \\
\hline AG102 & $\begin{array}{l}\triangle a c r A B \text { mutant } A G 100 \text {, owing } \\
\text { acrF gene markedly } \\
\text { over-expressed; TET }\end{array}$ \\
\hline MC4100 & Wild type E. coli \\
\hline W3110 & Wild type E. coli \\
\hline \multicolumn{2}{|c|}{ Enterobacter aerogenes } \\
\hline ATCC13048 & Reference strain \\
\hline CM64 & $\begin{array}{l}\mathrm{CHL}^{\mathrm{R}} \text { resistant variant obtained } \\
\text { from } A T C C 13048 \text { over-expressing } \\
\text { the } A \text { AcrAB pump }\end{array}$ \\
\hline EA3 & $\begin{array}{l}\text { Clinical MDR isolate; } C^{C H L^{R}}, N^{\prime} R^{R} \\
O F X^{R}, S P X^{R}, M O X^{R}, C F T^{R}, A^{R} M^{R}, F E\end{array}$ \\
\hline EA27 & $\begin{array}{l}\text { Clinical MDR isolate exhibiting } \\
\text { energy-dependent norfloxacin an } \\
\text { chloramphenicol efflux with KAN } \\
\text { AMP }^{R} \text { NAL }^{R} \text { STR }^{R} T^{2} T^{R}\end{array}$ \\
\hline EA289 & KAN sensitive derivative of EA27 \\
\hline EA294 & EA289 acrA::KAN ${ }^{R}$ \\
\hline EA298 & EA 289 tolC::KAN ${ }^{R}$ \\
\hline
\end{tabular}

Enterobacter cloacae

$\begin{array}{ll}\text { ECCI69 } & \text { Clinical MDR isolates, } \mathrm{CHL}^{\mathrm{R}} \\ \text { BM67 } & \text { Clinical MDR isolates, } \mathrm{CHL}^{\mathrm{R}} \\ \text { BM47 } & \text { Clinical MDR isolates, } \mathrm{CHL}^{\mathrm{R}}\end{array}$

Klebsiella pneumoniae

$\begin{array}{ll}\text { ATCC12296 } & \text { Reference strain } \\ \text { KP55 } & \text { Clinical MDR isolate, } \text { TET }^{R}, \text { AMP }^{R}, \\ & \text { ATM }^{R}, \text { CEF }^{R} \\ \text { KP63 } & \text { Clinical MDR isolate, } \text { TET }^{R,} \text { CHL }^{R}, \\ & \text { AMP }^{R,} \text { ATM }^{R} \\ \text { K24 } & \text { AcrAB-TolC, Laboratory collection } \\ & \text { of UNR-MD1, University of } \\ & \text { Marseille, France } \\ \text { K2 } & \text { AcrAB-TolC, Laboratory collection } \\ & \text { of UNR-MD1, University of } \\ & \text { Marseille, France }\end{array}$

Providencia stuartii

NEA16 Clinical MDR isolate, AcrAB-TolC

ATCC29916 Clinical MDR isolate, AcrAB-TolC

PS2636 Clinical MDR isolate, AcrAB-TolC

PS299645 Clinical MDR isolate, AcrAB-TolC

Pseudemonas aeruginosa
PA 01
Reference strain
PA 124
MDR clinical isolate

Table 2 Bacterial strains used in this study and their features (Continued)

\begin{tabular}{|c|c|c|c|}
\hline \multirow{3}{*}{ References } & \multicolumn{3}{|l|}{ (Contınued) } \\
\hline & \multicolumn{3}{|l|}{ S. aureus } \\
\hline & ATCC 25923 & Reference strain & \\
\hline [50] & MRSA 3 & $\begin{array}{l}\text { Clinical MDR isolate OFX } X^{R}, K^{\prime} N^{R} \text {, } \\
T^{R} T^{R}, E^{R} M^{R}\end{array}$ & [65] \\
\hline [50-52] & MRSA 4 & $\begin{array}{l}\text { Clinical MDR isolate OFX }{ }^{R}, K^{\prime} A N^{R} \text {, } \\
\mathrm{CHL}^{\mathrm{R}}, \mathrm{CIP}^{\mathrm{R}}\end{array}$ & \\
\hline$[53,54]$ & MRSA 6 & $\begin{array}{l}\text { Clinical MDR isolate OFX } X^{R}, F L X^{R}, \\
\text { KAN }^{R}, T^{R} T^{R}, C P^{R}, I M / C S^{R}\end{array}$ & \\
\hline$[55]$ & MRSA 8 & $\begin{array}{l}\text { Clinical MDR isolate OFX } X^{R}, F L X^{R} \text {, } \\
\operatorname{KAN}^{R}, \mathrm{ERM}^{R}, \mathrm{CIP}^{R}, I M / C S^{R}\end{array}$ & \\
\hline \multirow[t]{2}{*}[55,56]{} & MRSA 11 & $\begin{array}{l}\text { Clinical MDR isolate OFX } X^{R}, K^{\prime} A N^{R} \text {, } \\
\text { ERM }^{R}, C I P^{R}, I M / C S^{R}\end{array}$ & \\
\hline & MRSA 12 & $\begin{array}{l}\text { Clinical MDR isolate OFX } X^{R}, F L X^{R} \text {, } \\
\text { KAN }^{R}, E^{R} M^{R}, I M / C S^{R}\end{array}$ & \\
\hline$[58,59]$ & \multicolumn{3}{|c|}{$\begin{array}{l}\mathrm{AMP}^{\mathrm{R}}, \mathrm{ATM}^{\mathrm{R}}, \mathrm{CEF}^{\mathrm{R}}, \mathrm{CFT}^{\mathrm{R}}, \mathrm{CHL}^{\mathrm{R}}, \mathrm{CIP}^{\mathrm{R}}, \mathrm{ERM}^{\mathrm{R}}, \mathrm{FEP}^{\mathrm{R}}, \mathrm{FLX}^{\mathrm{R}}, \mathrm{IM} / \mathrm{CS}^{\mathrm{R}}, \mathrm{KAN}^{\mathrm{R}}, \mathrm{MOX}^{\mathrm{R}}, \\
\mathrm{OFX}^{\mathrm{R}}, \mathrm{STR}^{\mathrm{R}}, \mathrm{TET}^{\mathrm{R}}, \text { Resistance to ampicillin, aztreonam, cephalothin, cefadroxil, } \\
\text { chloramphenicol, Ciprofloxacin, Erythromycin, cefepime, Flomoxef, Imipenem/ } \\
\text { Cilastatin sodium, kanamycin, moxalactam, streptomycin, and tetracycline; } \\
\text { MDR multidrug resistant }\end{array}$} \\
\hline
\end{tabular}

shown in Table 4, extracts from leaves of Alstonia bonnei, Paullinia pinnata and Catharanthus roseus displayed wide spectra of activity in comparison to those from bark and stems of the same plants. The various plant extracts (when they were active) had MIC between 64 and $1024 \mu \mathrm{g} / \mathrm{mL}$. Leaves of Catharanthus roseus showed the best spectrum of activity, inhibiting the growth of $86.11 \%(31 / 36)$ of the bacteria (24/29 Gramnegative bacteria and 7/7 Gram-positive bacteria). The leaves extract of Croton macrostachys also had an interesting activity (30/36; $83.33 \%)$, followed by extract of the leaves of $P$. pinnata $(29 / 36 ; 80.55 \%)$ and the whole plant extract of $A$. conyzoides (25/36; $69.44 \%)$. The lowest MIC value of $64 \mu \mathrm{g} / \mathrm{mL}$ was obtained with the Paullinia pinnata stems extract and Cassia obtusifolia extract against the strain of Staphylococcus aureus MRSA8. In general, analysis of results shows that MBCs were obtained in $3.35 \%$ (7/209) of cases where plant extracts were active.

\section{Discussion}

Several classes of secondary metabolites such as alkaloids, triterpenes, sterols, flavonoids, polyphenols and saponins have been reported to have antibacterial properties [13-15]. Their presence in the studied plant extracts could explain the antibacterial effects of the tested samples. The need to find new molecules from medicinal plants with effective mechanisms of action against the multidrug-resistant phenotype is a necessity nowadays. All plants used in traditional medicine which have MIC values less than $8 \mathrm{mg} / \mathrm{mL}$ are considered active [16]. A plant extract has significant antibacterial 
Table 3 Extraction yields and phytochemical composition of the plant extracts

\begin{tabular}{|c|c|c|c|c|c|c|c|}
\hline \multirow[t]{2}{*}{ Plant extract (used part) } & \multirow[t]{2}{*}{ Extraction yield (\%) } & \multicolumn{6}{|c|}{ Phytochemicals groups } \\
\hline & & Alkaloids & Triterpenes & Sterols & Flavonoids & Polyphenols & Saponins \\
\hline A. boonei (leaves) & $15.8 \%$ & - & - & + & - & + & + \\
\hline A. boonei (bark) & $9.65 \%$ & + & + & + & + & + & + \\
\hline A. conyzoïdes (whole plant) & $8.52 \%$ & - & + & + & - & - & + \\
\hline C. macrostachys (Leaves) & $12.72 \%$ & - & + & + & - & + & + \\
\hline C. obtusifolia (whole plant) & $7.11 \%$ & + & + & + & + & + & + \\
\hline C. roseus (leaves) & $6.89 \%$ & + & - & + & + & + & + \\
\hline C. roseus (stem) & $4.23 \%$ & + & + & + & + & + & - \\
\hline P. pinnata (leaves) & $10.84 \%$ & - & + & + & - & + & + \\
\hline P. pinnata (stem) & $5.47 \%$ & - & + & + & - & + & + \\
\hline
\end{tabular}

+: presence; -: absence

activity if MIC is $100 \mu \mathrm{g} / \mathrm{mL}$, moderate if its $\mathrm{MIC}$ is between 100 and $625 \mu \mathrm{g} / \mathrm{mL}$ and low when MIC is above $625 \mu \mathrm{g} / \mathrm{mL}$ [17]. Based on the above criteria, it can be deduced that all tested plants had antibacterial activity as MIC values below $8 \mathrm{mg} / \mathrm{mL}$ were obtained with each extract on at least one bacterial strain. MIC values above $625 \mu \mathrm{g} / \mathrm{mL}$ were obtained with extract from A. boonei bark against 2/36 (5.5 \%) tested bacteria as well as with $C$. roseus stem extract against 6/36 (16.7\%) microorganisms tested, indicating that they rather displayed low antibacterial effects. Nonetheless, the activity obtained with the Paullinia pinnata stems extract and Cassia obtusifolia extract against the strain of Staphylococcus aureus MRSA8 (MIC value of $64 \mu \mathrm{g} / \mathrm{mL}$ ) could be considered important. Moderate activity was obtained in many cases. In fact, MIC values ranged from 128 to $512 \mu \mathrm{g} / \mathrm{mL}$ were obtained with extract from $A$. conyzoides (whole plant) against 12/36 (33.3\%) tested bacteria, $A$. boonei leaves against 19/36 (52.8\%), C. obtusifolia (whole plant) against $17 / 36(47.2 \%), C$. roseus leaves against 18/36 (50 \%), C. macrostachys (leaves) against 25/36 (69.4\%), and $P$. pinnata stem and leaves against 13/ 36 (36.1 \%) and 19/36 (52.8\%) respectively.

Though the antibacterial activities of some of the tested plants have already been reported, their effects against MDR phenotypes are being documented for the first time. The extract from the leaves of $C$. roseus had a broad antibacterial activity (31/36; $86.11 \%)$; Nayak and Pereira [18] and Kamaraj et al. [19] reported the antibacterial activity of this plant extract on some sensitive bacteria. Several alkaloids were isolated from this plant $[20,21]$ and these compounds could also be responsible for the antibacterial activity of this plant [22]. MIC values obtained with extract of leaves of $C$. macrostachys are between 128 and $1024 \mu \mathrm{g} / \mathrm{mL}$; Antibacterial compounds previously isolated from this plant include the triterpenoid, lupeol [23]. The extract of $P$. pinnata possessed a good activity (MIC of $64 \mu \mathrm{g} / \mathrm{mL}$ ) against $S$. aureus MRSA8 while the extract from the leaves was active against $80.55 \%$ (29/36) of the studied microorganisms. Lunga et al. [24] demonstrated the activity of this plant on strains of Salmonella $s p$. with a bacteriostatic effect, corroborating our findings. The extract of C. obtusifolia significantly inhibited the growth of $S$. aureus MRSA8 with MIC of $64 \mu \mathrm{g} / \mathrm{mL}$, and was active on 22 of the 36 tested microorganisms. The activity obtained in this study is much better than that mentioned by Doughari et al. [25]. In fact, they obtained MIC of $2000 \mu \mathrm{g} / \mathrm{mL}$ and $1000 \mu \mathrm{g} / \mathrm{mL}$ on clinical isolate of $S$. aureus and $P$. aeruginosa respectively. This could be due to the difference of phytochemical composition as the environmental conditions influence the availability as well as the amounts of some secondary metabolites in the plant. One of the best suited secondary metabolite from this plant is emodin (anthraquinone) which possesses a good antibacterial activity against $S$. aureus [26]; this could explain the interesting activity observed vis-à-vis of MRSA in this study. The extract of $A$. conyzoides had a relatively low activity on all studied microorganisms. Nevertheless, MIC of $256 \mu \mathrm{g} / \mathrm{mL}$ vis-a-vis E. aerogenes EA-CM64 and EA27, P. stuartii PS2636, S. aureus MRSA 4 which are multi-drug resistant clinical strains were obtained; this could explain the use of this plant in traditional medicine. Leaves and bark extracts of $A$. bonnei had a moderate activity against Gram-negative bacteria whilst bark extract was not active against Gram-positive species; this is explained by the fact that some antimicrobial compounds have specific activity spectrum (narrow) and therefore will not be active on certain categories or certain species of microorganisms [27]. Though the overall activity of the tested plants can be considered moderate, the results of this study are interesting taking in account the fact that most of the tested bacterial strains were MDR phenotypes. 
Table $4 \mathrm{MIC}$ and MBC (in bracket) of plant extracts and reference drugs

\begin{tabular}{|c|c|c|c|c|c|c|c|c|c|c|}
\hline & \multirow{2}{*}{$\begin{array}{l}\text { A. conyzoïdes } \\
\text { (whole plant) }\end{array}$} & \multicolumn{2}{|l|}{ A. boonei } & \multirow{2}{*}{$\begin{array}{l}\text { C. obtusifolia } \\
\text { (whole plant) }\end{array}$} & \multicolumn{2}{|l|}{ C. roseus } & \multirow{2}{*}{$\begin{array}{l}\text { C. macrostachys } \\
\text { (leaves) }\end{array}$} & \multicolumn{2}{|l|}{ P. pinnata } & \multirow{2}{*}{$\begin{array}{l}\text { Reference drugs } \\
\text { Chloramphenicol }\end{array}$} \\
\hline & & Leaves & Bark & & Leaves & Stem & & Leaves & (Stem) & \\
\hline \multicolumn{11}{|l|}{ Escherichia coli } \\
\hline ATCC8739 & - & $512(-)$ & - & - & $512(-)$ & - & $512(-)$ & $1024(-)$ & - & $2(128)$ \\
\hline ATCC10536 & $256(-)$ & - & - & - & $512(-)$ & $1024(-)$ & $256(-)$ & $128(-)$ & - & $<2(64)$ \\
\hline AG100 & $1024(-)$ & - & - & $256(1024)$ & $1024(-)$ & - & $1024(-)$ & $256(-)$ & $128(-)$ & $8(128)$ \\
\hline AG100A & $1024(-)$ & - & - & $512(-)$ & $128(-)$ & $256(-)$ & - & $256(-)$ & $256(-)$ & $<2(128)$ \\
\hline AG100A $A_{\text {TET }}$ & $1024(-)$ & $512(-)$ & $1024(-)$ & - & $1024(-)$ & - & - & - & $512(-)$ & $32(-)$ \\
\hline AG102 & $512(-)$ & $512(-)$ & $1024(-)$ & - & - & - & $1024(-)$ & - & $256(-)$ & $64(-)$ \\
\hline MC4100 & - & - & - & - & $512(-)$ & $512(-)$ & $256(-)$ & $256(-)$ & $1024(-)$ & $16(-)$ \\
\hline W3110 & $1024(-)$ & - & $128(-)$ & - & $512(-)$ & - & $256(-)$ & $1024(-)$ & - & $2(-)$ \\
\hline \multicolumn{11}{|c|}{ Pseudomonas aeruginosa } \\
\hline PA 01 & - & - & - & $256(-)$ & $512(-)$ & $256(-)$ & $256(-)$ & $256(-)$ & $1024(-)$ & $32(-)$ \\
\hline PA 124 & - & - & - & - & - & - & - & - & - & $128(-)$ \\
\hline \multicolumn{11}{|c|}{ Enterobacter aerogenes } \\
\hline ATCC13048 & $1024(-)$ & $512(-)$ & - & $512(-)$ & - & - & $128(-)$ & - & - & $4(32)$ \\
\hline EA-CM64 & $256(-)$ & - & - & $512(-)$ & $1024(-)$ & - & $256(-)$ & $512(-)$ & $1024(-)$ & $256(-)$ \\
\hline EA3 & - & - & - & - & $256(-)$ & $1024(-)$ & $128(-)$ & - & - & $256(-)$ \\
\hline EA27 & $256(-)$ & $512(-)$ & $512(-)$ & $512(1024)$ & $512(-)$ & $1024(-)$ & $512(-)$ & $512(-)$ & - & $32(-)$ \\
\hline EA289 & $512(-)$ & $1024(-)$ & $1024(-)$ & $256(-)$ & $512(-)$ & $1024(-)$ & $512(-)$ & $512(-)$ & $512(-)$ & $64(-)$ \\
\hline EA298 & $1024(-)$ & - & $1024(-)$ & $512(-)$ & $1024(-)$ & $1024(-)$ & $128(-)$ & $512(-)$ & $512(-)$ & 128 \\
\hline \multicolumn{11}{|c|}{ Providencia stuartii } \\
\hline NEA16 & $1024(-)$ & $512(-)$ & $1024(-)$ & 1024 & $1024(-)$ & - & $1024(-)$ & $1024(-)$ & $1024(-)$ & $32(256)$ \\
\hline ATCC29916 & $512(-)$ & $512(-)$ & - & - & - & - & $256(-)$ & $1024(-)$ & - & $16(256)$ \\
\hline PS2636 & $256(-)$ & - & - & - & - & $256(-)$ & $256(-)$ & $256(-)$ & - & $16(256)$ \\
\hline PS299645 & $1024(-)$ & $512(-)$ & - & - & $256(-)$ & $512(-)$ & $512(-)$ & $512(-)$ & - & $64(-)$ \\
\hline \multicolumn{11}{|c|}{ Klebsiella pneumoniae } \\
\hline ATCC11296 & $512(-)$ & $512(-)$ & $1024(-)$ & $1024(-)$ & $1024(-)$ & $1024(-)$ & $512(-)$ & $1024(-)$ & - & $8(256)$ \\
\hline KP55 & $512(-)$ & $512(-)$ & - & $256(-)$ & $512(-)$ & - & $256(-)$ & $1024(-)$ & $256(-)$ & $32(256)$ \\
\hline KP63 & $1024(-)$ & $1024(-)$ & - & $1024(-)$ & $512(-)$ & - & - & $1024(-)$ & $1024(-)$ & $32(-)$ \\
\hline K24 & $1024(-)$ & $512(-)$ & $1024(-)$ & $1024(-)$ & $1024(-)$ & - & $512(-)$ & $512(-)$ & - & $64(256)$ \\
\hline K2 & $1024(-)$ & $256(-)$ & - & $1024(-)$ & $512(-)$ & $512(-)$ & - & - & - & $8(256)$ \\
\hline
\end{tabular}


Table $4 \mathrm{MIC}$ and MBC (in bracket) of plant extracts and reference drugs (Continued)

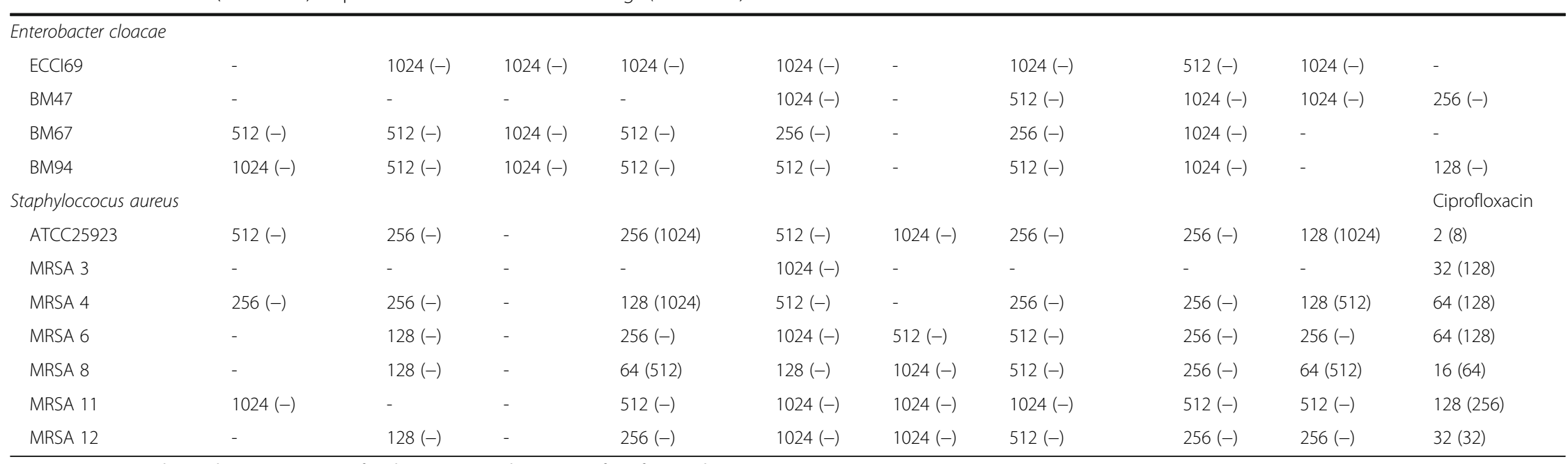




\section{Conclusion}

The present study demonstrates that plants studied and mostly C. macrostachys, C. roseus and $P$. pinnata contain phytochemicals with valuable antibacterial activities vis-à-vis multi-drug resistant phenotypes. They could be used in the management of bacterial infections including MDR phenotypes.

\section{Abbreviations \\ A. conyzoides: Ageratum conyzoides; Alstonia boonei: Alstonia boonei; ATCC: American type culture collection; C. macrostachys: Croton macrostachys; C. roseus: Catharanthus roseus; Cassia obtusifolia: Cassia obtusifolia; CFU: Colony forming unit; DMSO: Dimethylsufoxide; E. aerogenes: Enterobacter aerogenes; $E$. cloacae: Enterobacter cloacae; E. coli: Escherichia coli; INT: p-lodonitrotetrazolium chloride; K pneumoniae: Klebsiella pneumoniae; MBC: Minimal bactericidal concentration; MDR: Multi-drug resistant; MHA: Mueller Hinton Agar; MHB: Mueller Hinton Broth; MIC: Minimum inhibitory concentration; MRSA: Methicillin resistant Staphylococcus aureus; MSA: Mannitol Salt Agar; $P$. aeruginosa: Pseudomonas aeruginosa; $P$. pinnata: Paullinia pinnata; $P$. stuartii: Providencia stuartii; RND: Resistance nodulation cell division; $S$. aureus: Staphyloccocus aureus}

\section{Acknowledgements}

Authors are thankful to the Cameroon National Herbarium (Yaounde) for the plant identification. Authors are also thankful to UMR-MD1 (Mediterranean University, Marseille, France) and Dr Jean P. Dzoyem (University of Dschang) for providing some clinical bacteria.

\section{Funding}

No funding.

\section{Availability of data and materials}

The datasets supporting the conclusions of this article are presented in this main paper. Plant materials used in this study have been identified at the Cameroon National Herbarium where voucher specimens are deposited.

\section{Authors' contributions}

IKV carried out the study; IKV and VK designed the experiments and wrote the manuscript; VK and VPB supervised the work; VK provided the bacterial strains; all authors read and approved the final manuscript.

\section{Competing interests}

The authors declare that they have no competing interests.

\section{Consent for publication}

Not applicable in this section.

\section{Ethics approval and consent to participate}

Not applicable in this section.

\section{Author details}

'Department of Biochemistry, Faculty of Science, University of Dschang, P.O. Box 67, Dschang, Cameroon. ²Department of Biochemistry, Faculty of Science, University of Yaounde I, Yaounde, Cameroon.

Received: 12 August 2016 Accepted: 6 October 2016

Published online: 10 October 2016

\section{References}

1. Pop-Vicas A, Tacconelli E, Gravenstein S, Lu B, D'Agata EM. Influx of multidrug-resistant, gram-negative bacteria in the hospital setting and the role of elderly patients with bacterial bloodstream infection. Infect Control Hosp Epidemiol. 2009;30(4):325-31.

2. Garnacho-Montero J, Corcia-Palomo Y, Amaya-Villar R, Martin-Villen L. How to treat VAP due to MDR pathogens in ICU patients. BMC Infect Dis. 2014;14:135.

3. Vargiu VA, Ruggerone P, Opperman JT, Nguyen TS, Nikaido H. Molecular Mechanism of MBX2319 inhibition of Escherichia coli AcrB multidrug efflux pump and comparison with other inhibitors. Antimicrob Agents Chemother. 2014;58(10):6224-34.
4. Linhares I, Raposo T, Rodrigues A, Almeida A. Incidence and Diversity of Antimicrobial Multidrug Resistance Profiles of Uropathogenic Bacteria. Biomed Res Int. 2015;2015:354084.

5. Rice BL. Antimicrobial Resistance in Gram-Positive Bacteria. Am J Med. 2006; 119:11-9.

6. CDC. Antibiotic resistance threats in the United States. Atlanta: U.S. Department of Health and Human Services, CDC; 2013.

7. WHO. Traditional medicine. 2003. http://www.who.int/mediacentre/ factsheets/2003/fs134/en/. Accessed 20 June 2016.

8. FAO. Trade in medicinal plants. 2004. ftp://ftp.fao.org/docrep/fao/008/ af285e/af285e00.pdf. Accessed 20 June 2016.

9. Kuete V, Metuno R, Ngameni B, Tsafack AM, Ngandeu F, Fotso GW, et al. Antimicrobial activity of the methanolic extracts and compounds from Treculia obovoidea (Moraceae). J Ethnopharmacol. 2007;112:531-6.

10. Tchinda FC, Voukeng Kl, Penlap BV, Kuete V. Antibacterial activities of the methanol extracts of Albizia adianthifolia, Alchornea laxiflora, Laportea ovalifolia and three other Cameroonian plants against multi-drug resistant Gram-negative bacteria. Saudi J Biol Sci. 2016. doi:10.1016/j.sjbs.2016.01.033.

11. Mambe TF, Voukeng Kl, Penlap BV, Kuete V. Antibacterial activities of methanol extracts from Alchornea cordifolia and four other Cameroonian plants against MDR phenotypes. J Taibah Univ Med Sci. 2016;11(2):121-7.

12. Harborne JB. Phytochemical Methods. New York: Chapman and Hall; 1973.

13. Kuete V, Ngameni B, Simo CC, Tankeu RK, Ngadjui BT, Meyer J, Lall N, Kuiate JR. Antimicrobial activity of the crude extracts and compounds from Ficus chlamydocarpa and Ficus cordata (Moraceae). J Ethnopharmacol. 2008; 120(1):17-24

14. Cowan MM. Plant products as antimicrobial agents. Clin Microbiol Rev. 1999:12(4):564-82.

15. Mbaveng TA, Sandjo LP, Tankeo SB, Ndifor AR, Pantaleon A, Nagdjui TB, Kuete $V$. Antibacterial activity of nineteen selected natural products against multi-drug resistant Gram-negative phenotypes. SpringerPlus. 2015;4:823.

16. Fabry W, Okemo PO, Ansorg R. Antibacterial activity of East African medicinal plants. J Ethnopharmacol. 1998;60:79-84.

17. Kuete V. Potential of Cameroonian plants and derived-products against microbial infections: A review. Planta Med. 2010;76:1479-91.

18. Nayak BS, Pereira PLM. Catharanthus roseus flower extract has wound-healing activity in Sprague Dawley rats. BMC Complement Altern Med. 2006;6:41.

19. Kamaraj C, Rahuman AA, Siva C, Iyappan M, Kirthi VA. Evaluation of antibacterial activity of selected medicinal plant extracts from south India against human pathogens. Asian Pac J Trop Biomed. 2012;2(1):296-301.

20. Goyal P, Khanna A, Chauhan A, Chauhan G, Kaushik P. In vitro evaluation of crude extracts of Catharanthus roseus for potential antibacterial activity. Int J Green Pharm. 2008;2(3):176-81.

21. Almagro L, Fernandez-Perez F, Pedreno MA. Indole alkaloids from Catharanthus roseus: bioproduction and their effect on human health. Molecules. 2015;20:2973-3000.

22. Ali AMA, Lafta HA, Jabar HKS. Antibacterial activity of alkaloidal compound isolated from leaves of Catharanthus roseaus (L.) against multi-drug resistant strains. Res Pharm Biotech. 2014;5(2):13-21.

23. Obey KJ, von Wright A, Orjala J, Kauhanen J, Tikkanen-Kaukanen C. Antimicrobial activity of Croton macrostachyus stem bark extracts against several human pathogenic bacteria. J Pathog. 2016;2016:1453428.

24. Lunga KP, Tamokou JDD, Fodouop CSP, Kuiate JR, Tchoumboue J, Gatsing D. Antityphoid and radical scavenging properties of the methanol extracts and compounds from the aerial part of Paullinia pinnata. Springerplus. 2014;3:302.

25. Doughari $\mathrm{JH}$, El-mahmood AM, Tyoyina I. Antimicrobial activity of leaf extracts of Senna obtusifolia (L). Afr J Pharm Pharmacol. 2008;2(1):7-13.

26. Zhou L, Yun BY, Wang YJ, Xie MJ. Antibacterial mechanism of emodin on Staphylococcus aureus. Chin J Biochem Mol Biol. 2011;27(12):1156-60.

27. Yamamoto T, Matsui H, Yamaji K, Takahashi T, Overby A, Nakamura M, Matsumoto A, Nonaka K, Sunazuka T, Omura S, Nakano H. Narrowspectrum inhibitors targeting an alternative menaquinone biosynthetic pathway of Helicobacter pylori. J Infect Chemother. 2016. doi:10.1016/j. jiac.2016.05.012

28. Majekodunmi SO, Adegoke OA, Odeku OA. Formulation of the extract of the stem bark of Alstonia boonei as tablet dosage form. Trop J Pharm Res. 2008:7(2):987-94.

29. Adotey JPK, Adukpo GE, Boahen YO, Armah FA. A review of the ethnobotany and pharmacological importance of Alstonia boonei De wild (Apocynaceae). ISRN Pharmacol. 2012. doi:10.5402/2012/587160. 
30. Bello IS, Oduola T, Adeosun OG, Omisore NOA, Raheem GO, Ademosun AA. Evaluation of Antimalarial Activity of Various Fractions of Morinda lucida Leaf Extract and Alstonia boonei stem Bark. Global J Pharmacol. 2009;3(3):163-65.

31. Akinmoladun CA, Ibukun EO, Afor E, Akinrinlola BL, Onibon TR, Akinboboye AO, Obuotor EM, Farombi EO. Chemical constituents and antioxidant activity of Alstonia boonei. Afr J Biotechnol. 2007;6(10):1197-201.

32. Olajide OA, Awe OS, Makinde MJ, Ekhelar IA, Olusola A, Morebise O, Okpako TD. Studies on the anti-inflammatory, antipyretic and analgesic properties of Alstonia boonei stem bark. J Ethnopharmacol. 2000;71:179-86.

33. Ferreres F, Pereira DM, Valentao P, Andrade PB, Seabra RM, Sottomayor M. New phenolic compounds and antioxidant potential of Catharanthus roseus. J Agric Food Chem. 2008;56(21):9967-74.

34. Mustafa RN, Verpoorte R. Phenolic compounds in Catharanthus roseus. Phytochem Rev. 2007;6:243-58

35. Okunade AL. Ageratum conyzoides L. (Asteraceae). Fitoterapia. 2002;73(1):1-16.

36. Lavergne R. Tisaneurs et Plantes Médicinales Indigènes de La Réunion. Saint Denis de La Réunion: Orphie; 2001.

37. Jonville MC, Kodja H, Strasberg D, Pichette A, Ollivier E, Frederich M, Angenot L, Legault J. Antiplasmodial, anti-inflammatory and cytotoxic activities of various plant extracts from the Mascarene Archipelago. J Ethnopharmacol. 2011;136:525-31.

38. Kuete V, Voukeng Kl, Tsobou R, Mbaveng TA, Wiench B, Penlap BV, Efferth T. Cytotoxicity of Elaoephorbia drupifera and other Cameroonian medicinal plants against drug sensitive and multidrug resistant cancer cells. BMC Complement Altern Med. 2013;13:250.

39. Bantie L, Assefa S, Teklehaimanot T, Engidawork E. In vivo antimalarial activity of the crude leaf extract and solvent fractions of Croton Macrostachyus Hocsht. (Euphorbiaceae) against Plasmodium berghei in mice. BMC Complement Altern Med. 2014;14:79.

40. Salatino A, Salatino FML, Negri G. Traditional uses, Chemistry and Pharmacology of Croton species (Euphorbiaceae). J Braz Chem Soc. 2007;18(1):11-33.

41. Kalayou S, Haileselassie M, Gebre-Egziabher G, Tikue T, Sahle S, Taddele H, Ghezu M. In-vitro antimicrobial activity screening of some ethnoveterinary medicinal plants traditionally used against mastitis, wound and gastrointestinal tract complication in Tigray Region, Ethiopia. Asian Pac J Trop Biomed. 2012;2(7):516-22

42. Kapingu MC, Guillaume D, Mbwambo HZ, Moshi JM, Uliso CF, Mahunnah RLA. Diterpenoids from the roots of Croton macrostachys. Phytochemistry. 2000;54(8):767-70

43. Tala FM, Tan NH, Ndontsa BL, Tane P. Triterpenoids and phenolic compounds from Croton macrostachyus. Biochem Syst Ecol. 2013;51:138-41.

44. Dave H, Ledwani L. A review on anthraquinones isolated from Cassia species and their applications. Indian J Nat Prod Resour. 2012;3(3):291-319.

45. Yang YC, Lim MY, Lee HS. Emodin isolated from Cassia obtusifolia (Leguminosae) seed shows larvicidal activity against three mosquito species. J Agric Food Chem. 2003;51(26):7629-31.

46. Yun-Choi HS, Kim JH, Takido M. Potential inhibitors of platelet aggregation from plant sources, v. anthraquinones from seeds of Cassia obtusifolia and related compounds. J Nat Prod. 1990;53(3):630-33.

47. Ju MS, Kim HG, Choi JG, Ryu JH, Hur J, Kim YJ, Oh MS. Cassiae semen, a seed of Cassia obtusifolia, has neuroprotective effects in Parkinson's disease models. Food Chem Toxicol. 2010;48(8-9):2037-44.

48. Miemanang $\mathrm{R}$, Krohn $\mathrm{K}$, Hussain $\mathrm{H}$, Dongo E. Paullinoside $\mathrm{A}$ and paullinomide $A$ : a new cerebroside and a new ceramide from leaves of Paullinia pinnata. Z Naturforsch. 2006;61:1123-27.

49. Okpekon T, Yolou S, Gleye C, Roblot F, Loiseau P, Bories C, Grellier P, Frappier F, Laurens A, Hocquemiller R. Antiparasitic activities of medicinal plants used in Ivory Coast. J Ethnopharmacol. 2004:90(1):91-7.

50. Viveiros M, Jesus A, Brito M, Leandro C, Martins M, Ordway D, Molnar AM, Molnar J, Amaral L. Inducement and reversal of tetracycline resistance in Escherichia coli K-12 and expression of proton gradient-dependent multidrug efflux pump genes. Antimicrob Agents Chemother. 2005;49:3578-82.

51. Kuete V, Ngameni B, Tangmouo JG, Bolla JM, Alibert-Franco S, Ngadjui BT, Pages JM. Efflux pumps are involved in the defense of Gram-negative bacteria against the natural products isobavachalcone and diospyrone. Antimicrob Agents Chemother. 2010;54:1749-52.

52. Okusu $H, M a D$, Nikaido $H$. AcrAB efflux pump plays a major role in the antibiotic resistance phenotype of Escherichia coli multiple-antibioticresistance (Mar) mutants. J Bacteriol. 1996:178:306-8.

53. Elkins CA, Mullis LB. Substrate competition studies using whole-cell accumulation assays with the major tripartite multidrug efflux pumps of Escherichia coli. Antimicrob Agents Chemother. 2007;51:923-9.
54. Kuete V, Alibert-Franco S, Eyong KO, Ngameni B, Folefoc GN, Nguemeving JR, Tangmouo JG, Fotso GW, Komguem J, Ouahouo BM, Bolla JM, Chevalier J, Ngadjui BT, Nkengfack AE, Pages JM. Antibacterial activity of some natural products against bacteria expressing a multidrug-resistant phenotype. Int J Antimicrob Agents. 2011;37:156-61

55. Baglioni P, Bini L, Liberatori S, Pallini V, Marri L. Proteome analysis of Escherichia coli W3110 expressing an heterologous sigma factor. Proteomics. 2003;3:1060-65.

56. Sar C, Mwenya B, Santoso B, Takaura K, Morikawa R, Isogai N, Asakura Y, Toride $Y$, Takahashi J. Effect of Escherichia coli wild type or its derivative with high nitrite reductase activity on in vitro ruminal methanogenesis and nitrate/nitrite reduction. J Anim Sci. 2005:83:644-52.

57. Ghisalberti D, Masi M, Pages JM, Chevalier J. Chloramphenicol and expression of multidrug efflux pump in Enterobacter aerogenes. Biochem Biophys Res Commun. 2005;328:1113-8.

58. Mallea M, Chevalier J, Bornet C, Eyraud A, Davin-Regli A, Bollet C, Pages JM. Porin alteration and active efflux: two in vivo drug resistance strategies used by Enterobacter aerogenes. Microbiology. 1998;144:3003-9.

59. Mallea M, Mahamoud A, Chevalier J, Alibert-Franco S, Brouant P, Barbe J, Pages JM. Alkylaminoquinolines inhibit the bacterial antibiotic efflux pump in multidrug-resistant clinical isolates. Biochem J. 2003;376:801-5.

60. Pradel E, Pages JM. The AcrAB-TolC efflux pump contributes to multidrug resistance in the nosocomial pathogen Enterobacter aerogenes. Antimicrob Agents Chemother. 2002;46:2640-43.

61. Voukeng IK, Kuete V. Epices Camerounaises et Bactéries multi-résistantes, Volume 1, Activités Biologiques et Synergie avec les Antibiotiques. Éditions universitaires européennes. 2013.

62. Chevalier J, Pages JM, Eyraud A, Mallea M. Membrane permeability modifications are involved in antibiotic resistance in Klebsiella pneumoniae. Biochem Biophys Res Commun. 2000;274:496-9.

63. Tran QT, Mahendran KR, Hajjar E, Ceccarelli M, Davin-Regli A, Winterhalter M Weingart $\mathrm{H}$, Pages JM. Implication of porins in beta-lactam resistance of Providencia stuartii. J Biol Chem. 2010;285:32273-81.

64. Lorenzi V, Muselli A, Bernardini AF, Berti L, Pages JM, Amaral L, Bolla JM. Geraniol restores antibiotic activities against multidrug-resistant isolates from gram-negative species. Antimicrob Agents Chemother. 2009:53:2209-11.

65. Paudel A, Hamamoto H, Kobayashi Y, Yokoshima S, Fukuyama T, Sekimizu K. Identification of novel deoxyribofuranosyl indole antimicrobial agents. Antibiot. 2012;65:53-7.

\section{Submit your next manuscript to BioMed Central and we will help you at every step:}

- We accept pre-submission inquiries

- Our selector tool helps you to find the most relevant journal

- We provide round the clock customer support

- Convenient online submission

- Thorough peer review

- Inclusion in PubMed and all major indexing services

- Maximum visibility for your research

Submit your manuscript at www.biomedcentral.com/submit
) Biomed Central 